\title{
Developing Retina and PNS Segments for Transplantation Into the Adult Host Eye: Reconstruction of the Mammalian Visual System. 2. Results
}

\author{
Brian H. Hallas, Ph.D., Howard F. Lowe, Glenn Jacobsen, Michael LaCorte, \\ Simone P. Lee and Michael F. Zanakis, Ph.D.* \\ Department of Anatomy and Neuroscience, New York College of Osteopathic Medicine, Old Westbury, NY \\ 11568 , and $*$ Department of Bioengineering, School of Engineering, The Cooper Union, \\ 51 Astor Place, New York, NY 10003, U.S.A.
}

\begin{abstract}
SUMMARY
The previous companion paper detailed a technique which allowed embryonic retinal ganglion cell axons to grow from the anterior eye chamber across a PNS bridge, and enter the adult host forebrain. Embryonic eyes of E11, E14, E18 and E21 animals were sutured to a PNS bridge, the embryonic eye implanted into an adult host eye, and the distal end of the bridge implanted into the host forebrain. Results indicate that when eyes of all ages are used for implantation, axons could be observed to grow from the embryonic retina, through the bridge and into the adult host forebrain. The axons extend for long distances in the host brain, reach various layers of the cortex and in a few animals enter the caudate/putamen complex. Control studies show that the bridge is used exclusively as the conduit to the brain, as opposed to the degenerated host optic nerve. Thus, the results presented in this paper indicate that successful grafting and transplantation is possible
\end{abstract}

Reprint address:

Brian H. Hallas, Ph.D.

Department of Anatomy and Neuroscience,

New York College of Osteopathic Medicine

Old Westbury, NY 11568

U.S.A. using the aforementioned technique. The results suggest that the described visual system reconstruction technique can be used for the study of development and transplantation in this system.

\section{KEY WORDS}

Embryonic, Transplantation, Retina, Regeneration

\section{INTRODUCTION}

The previous companion report described a new technique for the study of CNS reconstruction following visual system damage. That study outlined the feasibility of utilizing transplanted embryonic retinal ganglion cells to regenerate through an adult peripheral nerve bridge and into the adult CNS. This was accomplished by attaching an entire embryonic eye (of varying ages) to a segment of peripheral nerve. The peripheral nerve served as a bridge between the embryonic retinal ganglion cells and the adult host brain. The embryonic eye with the attached segment of peripheral nerve was then implanted into the adult host eye. The distal end of the peripheral nerve bridge was inserted into the host forebrain. This preparation allows for the tracing of fibers from the implant to the host 
CNS in a manner that is both unambiguous and reproducible.

The results of that study demonstrated that while the preparation is difficult to perfect, it does allow one to study a flexible yet well defined combination of systems. The system is capable of supporting the development of the embryonic system, and enabling growth of its retinal ganglion cell axons across the bridge and into the adult host's forebrain.

Thus, this paper reports on the findings of this experimental paradigm. Specifically, the preparation described previously was used, and after 30 to 180 days, growth of the embryonic system through the bridge and into the host CNS was evaluated histologically. This was accomplished by tracing the system with HRP both anterogradely and retrogradely. These experiments allow us to determine the uses and limitations of the technique by tracing the origin of the axons; e.g., whether they were from the implant or the host retinal ganglion cells.

\section{MATERIALS AND METHODS}

\section{Experiments}

Experiments were performed to determine 1) whether embryonic axons are capable of growing into a PNS bridge, and 2) if there is a preference for the adult (host) optic nerve or the bridge. The basic method used has been reported in the companion paper. In each of the experiments, the methods described for embryonic eye removal, tibial nerve removal, transplant preparation, and implantation were identical.

\section{Implantation of Embryonic Eye/PNS Bridge,} Optic Nerve Cut: In all animals $(\mathrm{N}=48)$, the embryonic eye was sutured to the PNS bridge as described earlier. The portion of the bridge with the embryonic eye attached was inserted into the host eye, and the other end inserted into the forebrain. Simultaneously, the host optic nerve was completely transected.

In one third of these animals, two days prior to the sacrifice times of 30 to 180 days postimplantation, HRP was applied to the transected bridge as described previously. Analysis of the HRP material yielded information about the growth of axons between the implant and the forebrain; i.e., whether the axons of the implant reached the forebrain, and the characteristics of the labelling pattern.

In another third of these animals, HRP was injected intraocularly two days prior to the 30 to 180 day sacrifice time point. HRP analysis determined the extent and configuration of axonal projection into the host CNS.

In the final third of these animals, HRP was injected into the forebrain and the entire eye (both host and implant) was examined for HRP labelled cells.

Embryonic Eye Implant, No PNS Bridge, Optic Nerve Cut: Another evenly divided group of animals (as above) received optic nerve transection, followed by an implant of embryonic eye without the attached PNS bridge. Prior to sacrifice, HRP was intraocularly injected in order to trace possible anterogradely regrown axons. The optic nerve, lateral geniculate body, superior colliculus and forebrain were analyzed histologically for presence of label. The purpose of this animal group was to determine whether the embryonic retinal ganglion cells could project to the host brain through the damaged optic nerve. Data from this group of animals yielded information regarding the growth potential of the embryonic retinal axons through a lesioned area.

PNS Bridge, No Embryonic Eye Implant, Optic Nerve Cut: In another group of animals divided as above, the hosts received an implant of the PNS bridge alone (the embryonic eye was not attached). The optic nerve was transected during the implant. Two days prior to sacrifice, animals were intraocularly injected with HRP. This allowed the determination of whether the host retinal ganglion cells were "rescued" and able to reorganize their axons into the peripheral nerve bridge. The optic nerve, optic tract, lateral 
geniculate body, PNS bridge, forebrain and superior colliculus were analyzed for label.

\section{Labelling Methods}

\section{Anterograde Labelling of the Forebrain from} the Retina and Bridge: Animals received anterograde labelling of the forebrain by either HRP injection into the host eye or into the bridge. For intraocular injections, animals received a $40 \%$ solution of free HRP (Sigma type VI) in water. A Kopf microinjector was used to pressure inject $3 \mu \mathrm{l}$ of solution intraocularly over a period of 30 minutes. Labelling of the bridge and forebrain will result using this technique.

In animals that received a bridge application, the skin over the bridge was excised over the skull, with care taken not to damage the bridge. The bridge appeared shiny white against the darker fascia, and was easily identifiable. With a No. 10 scalpel, the bridge was transected $2-3 \mathrm{~mm}$ from the burr hole. Gel-foam soaked HRP was applied directly to the transection site for 50 minutes. The Gel-foam was then removed and the stumps of the bridge were sealed with agar to prevent leakage of the HRP. The skin was then closed with wound clips over the bridge.

Retrograde Labelling of the Retina from the Bridge and Forebrain: In a third group of animals, and for each embryonic age group, $1-2 \mu \mathrm{L}$ of a $40 \%$ solution of HRP (Sigma type VI) was injected into the forebrain at the site where the distal portion of the tibial nerve bridge entered the cerebral cortex. This was accomplished by carefully drilling away the cranium surrounding the bridge and making a series of small injections $360^{\circ}$ around the bridge. After the injections were completed, Gel-foam was placed around the bridge, the skin over the bridge sutured closed and the animal sacrificed 48 hours later.

All histological procedures were performed as outlined in the previous report. They were carried out in a blinded fashion, without prior knowledge of the treatment group. Briefly, each animal was perfused transcardially with a $1 \%$ paraformaldehyde, $2.5 \%$ gluteraldehyde and $4 \%$ sucrose fixative. The eye, proximal and distal bridge segment and brain were then removed and sectioned at $50 \mu \mathrm{m}$ on a freezing microtome. Resulting sections were processed according to the tetramethylbenzidine (TMB) protocol of Mesulam /14/. Alternate sections were mounted, coverslipped and counterstained with thionin. For retrograde analyses, the total number of labelled ganglion cells of the host and donor retina were counted. The whole mounted retinae were processed for HRP histochemistry which established the quantity and distribution of retinal ganglion cells that projected through the bridge. For anterograde analyses, axonal growth towards the forebrain was determined by the presence of label in the bridge or forebrain.

\section{RESULTS}

In all host animals that had been implanted with E11, E14, E18 or E21 day eyes and no bridge, no evidence of HRP transport was observed at any sacrifice time. In most animals of this group, the embryonic eyes were found to have survived implantation and to have grown and differentiated into somewhat laminar structures that were reminiscent of normal retinae. However, no axons from these implant eyes grew into the lesioned host optic nerve, nor did any of the host retinal ganglion cells grow past the lesion. In fact, most of the host retinal ganglion cells appeared atrophied.

Likewise, when a peripheral nerve bridge was implanted into the host eye with no attached embryonic eye no evidence of axonal transport of HRP could be observed. The HRP that was injected into the host eye stayed in the host eye with no label being observed in the frontal cortex or in any part of the bridge. Since the host optic nerve had been transected at implantation, this suggests that the host's retinal ganglion cells axons did not grow into the bridge.

This is in contrast to the findings when an embryonic eye was sutured to a peripheral nerve 


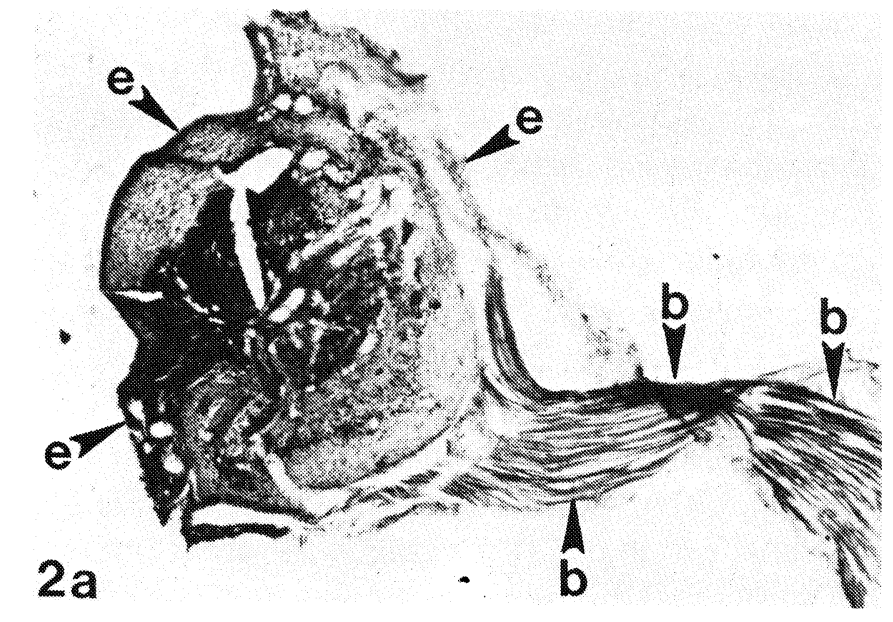

Fig. 2a: Low power photomicrograph of $E 18$ eye 90 days post-implantation in which HRP was injected intraocularly. Note the heavy label in the implant ("e" arrow) and bridge ("b" arrow).

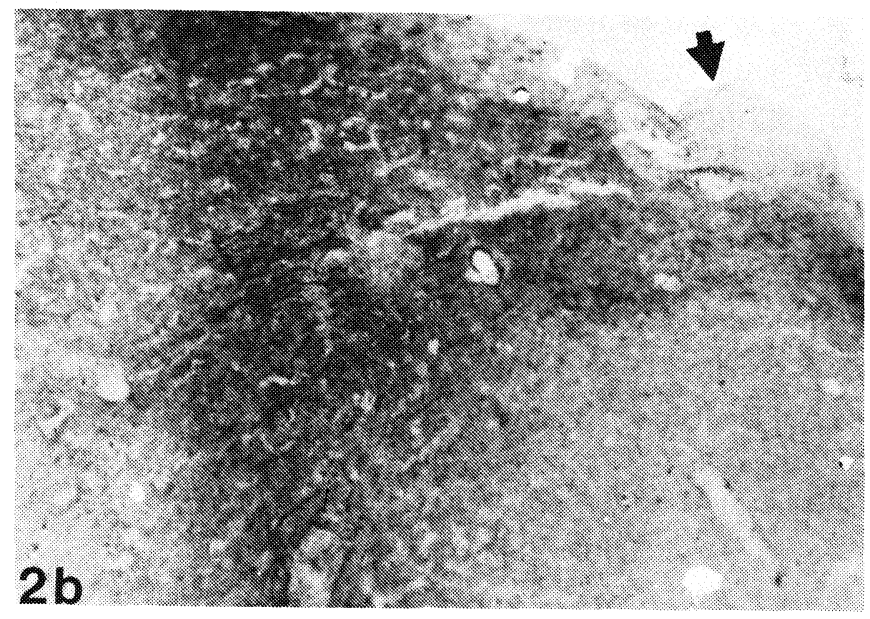

Fig. 2b: Photomicrograph of the terminal label in frontal cortex following HRP injection demonstrated in Figure 2a. Arrow denotes nerve bridge entering the cortex.

contained cells that appeared analogous to the normal retina. However, a true laminar arrangement could not be demonstrated throughout the entire retina. In many of these successful eye/bridge implants, a lens could also be observed.

\section{DISCUSSION}

In lower vertebrates such as the goldfish, damage to the optic nerve will result in survival of retinal ganglion cells, and regeneration of axons to their target (the optic tectum) to form functional contacts (e.g., appropriate synapses leading to restoration of visual function /9/. A significant reduction of retinal ganglion cells also occurs in amphibians /13/. In frogs, only approximately one-third of the retinal ganglion cells are present six months after optic nerve crush /17,22/. In neonatal mammals and avian species, there is an almost complete depletion of retinal ganglion cells following damage (interruption) of the optic nerve $/ 1,15,16 /$. Damage to the adult mammalian optic nerve will result in an aborted attempt to regenerate, and no new synapses will form on the target neurons from the retinal ganglion cells $/ 1,7,10,16 /$.

In an effort to stimulate regeneration, a number of experiments have focused on grafting CNS tissue from various parts of the fetal neuraxis into the brains of neonatal and adult animals. These cells can grow substantially, and survive for long periods of time, and differentiate to the extent that such grafts exhibit some topographical features of the mature, intact CNS (see /21/ for review). Most importantly, neuroanatomical studies have demonstrated afferent and efferent axonal projections between host and donor tissues, and electrophysiological analyses have demonstrated that donor implants can form functional synapses with the host brain, and can even mitigate functional deficits resulting from injury, aging, or congenital anomalies (see $/ 3 /$ for review).

Del Cerro et al $/ 4,5 /$ have found that developing retinas of various ages can survive and differentiate when transplanted into the anterior chamber of a rat of a different strain. For example, when a retina is transplanted into an albino host, the pigmented retina of the donor survives, differentiates, is well vascularized and can be distinguished from the host. In the vitreal chamber, fetal retina can also survive and differentiate $/ 4,24 /$. When the neonatal retina is transplanted into the host, the typical pattern formation is observed, and the graft integrates quite well with the host $/ 4,23 /$.

Mammalian neurons from various portions of 
the CNS have the capability to elongate their axons when a peripheral nerve graft is used as the growth substrate (see /26/ for review). Of course, axons of the CNS can originate from the regrowth of interrupted axons, sprouting of spared (undamaged) fibers, or elongation of collateral branches of injured neurons. Thus, in any study where regeneration into a graft may be observed, these possibilities must be considered. This is why axotomy is necessitated in regeneration studies, and why the optic nerve has been used successfully for such work. The axons of the retinal ganglion cells form the matrix of the optic nerve, and their origin is in the retina. Thus, transection of the optic nerve will ensure that those axons are disrupted. Using this model, a PNS bridge can be apposed to the severed optic nerve, and a relatively simple procedure can be performed to determine if axons found in the bridge originated from the retina. Indeed, this is the logic followed by Aguayo and his colleagues. Briefly, it has been determined that when the optic nerve is cut and a PNS bridge is sutured to the stump, the cut ganglion cell axons enter and grow through the bridge (see /2/ for review). Thus, the optic nerve has been replaced by a PNS graft in this preparation. Vidal-Sanz et al /25/ have used this technique as a bridge between the host eye and the mesencephalon. Interestingly, it has been shown that neurites of the fetal CNS will not extend into grafts of adult optic nerve in vitro /19/. This result may be related to the nonpermissive substrate properties of adult oligodendrocytes /18/. However, in other studies the environment of the adult optic nerve could be stimulated to elicit a limited regenerative response following injury. This was accomplished by the application of "growth associated triggering factors" derived from developing optic nerves $/ 11,12,20 /$.

In the previous companion paper we detailed the technique for mammalian visual system reconstruction. The data presented in this paper show that use of the technique can indeed yield results which are a first step in achieving this goal. Although this preparation may be considered an attempt at visual system reconstruction (where there has been damage to the host retina and optic nerve), other basic neurobiological issues can be explored. These include the determination of whether the embryonic axons can not only course through a PNS environment, but successfully enter and synapse on adult CNS targets. Also, the extent and architectural character of pattern formation can be determined since two time points are chosen. Other issues such as the contribution of the host eye to cell survivability, the contribution of implanted embryonic retina to a damaged host visual system and the effect of the implant on an intact visual system are the subject of this report.

In conclusion, the results demonstrate the technical feasibility of using this preparation in studying "visual system reconstruction". Further, these experiments support the contention that the embryonic retinal ganglion cells can grow long distances into host CNS tissue. The effect of various age embryonic eyes, and the effect of using other CNS targets such as the superior colliculus is currently being examined. Additionally, the contribution of the implant to the host system, as well as the contribution of the host environment to the developing system, will be determined in future studies.

\section{ACKNOWLEDGEMENTS}

This work was supported by grants from the Riland Neuromuscular Institute (BHH), and the New York State Science and Technology Foundation (MFZ).

\section{REFERENCES}

1. Allcutt D, Berry M, Sievers J. A quantitative comparison of the reactions of retinal ganglion cells to optic nerve crush in neonatal and adult mice. Dev Brain Res 1984; 16: 219-223.

2. Aguayo AJ, Vidal-Sanz M, Villegas-Perez MP, Bray GM. Growth and connectivity of axotomized retinal neurons in adult rats with optic nerves substituted by 
PNS grafts linking the eye and the midbrain. Ann NY Acad Sci 1987; 495: 1-9.

3. Bjorklund A, Stenevi U. Intracerebral neural implants: Neuronal replacement and reconstruction of damaged circuitries. Ann Rev Neurosci 1984; 7: 279-308.

4. del Cerro M. Gash DM, Rao GN. Notter MF, Wiegand SJ, Sathi S, del Cerro C. Retinal transplants into the anterior chamber of the rat eye. Neurosci 1987; 21: 707-723.

5. del Cerro M, Gash DM, Rao GN. Retinal transplants into normal and damaged adult retinas. Soc Neurosci Abstr 1987; 11: Part 1, 15.

6. del Cerro M, Gash DM, Rao GN, Notter MF, Wiegand SJ, Guipta M. Intraocular retinal transplants. Invest Ophthalmol 1985; 26: 1182-1185.

7. Gan SK, Harvey AR. Lack of ingrowth of retinal axons into the visually deafferented superior colliculus of young rats: A horseradish peroxidase study. Neurosci Lett 1986; 70: 10-16.

8. Greene HSN. The use of transplanted tissues in biology and histology. In: Bourne GH, (Ed), In Vivo Techniques in Histology. Baltimore: Williams and Wilkins, 1967; pp 80-112.

9. Grafstein B, Forman DS. Intracellular transport in neurons. Physiol Rev 1980; 60: 1167-1283.

10. Grafstein B, Ingoglia NA. Intracranial transection of the optic nerve in adult mice: preliminary observations. Exp Neurol 1982; 76: 318-330.

11. Hadani M, Harel A, Solomon A, Belkin M, Lavie V, Schwartz M. Substances from the optic nerve of neonatal rabbit induce regeneration-associated responses in the injured optic nerve of adult rabbit. Proc Natl Acad Sci USA 1984; 81:7965-7969.

12. Humphrey MF, Beazley LD. Retinal ganglion cell death during optic nerve regeneration in the frog Hyla moorei. J Comp Neurol 1985; 236: 382-402.

13. Lavie V, Harel A, Doron A, Solomon A, Lobel D, Belkin M, Ben-Basat S, Sharma S, Schwartz M. Morphological response of injured adult rabbit optic nerve to implants containing media conditioned by growing optic nerves. Brain Res 1987; 419: 166-172.

14. Mesulam M-M. Tetramethylbenzidine for horseradish peroxidase neurohistochemistry: A noncarcinogenic blue reaction product with superior sensitivity for visualizing neural afferents and efferents. J Histochem Cytochem 1978; 26: 106-117.

15. Muchnick N, Hibbard E. Avian retinal ganglion cells resistant to degeneration after optic nerve lesion. Exp Neurol 1980; 68: 205-216.

16. Muchnick-Miller N, Oberdorfer M. Neuronal and neuroglial responses following retinal lesions in the neonatal rat. J Comp Neurol 1981; 202: 493-504.

17. Scalia F, Arango V, Singman EL. Loss and displacement of ganglion cells after optic nerve regeneration in adult Rana pipiens. Brain Res 1985; 344: 267-280.

18. Schwab ME, Caroni P. Oligodendrocytes and CNS myelin are nonpermissive substrates for neurite growth and fibroblast spreading in vitro. J. Neurosci 1988; 8: 2381-2393.

19. Schwab ME, Thoenen $H$. Dissociated neurons regenerate into sciatic but not optic nerve explants in culture irrespective of neurotrophic factors. J Neurosci 1985; 5: 2415-2423.

20. Schwartz M, Belkin M, Harel A, Solomon A, Lavie V, Hadani M, Rachailovich I, Stein-Izsak C. Regenerating fish optic nerves and regeneration-like response in injured optic nerves of adult rabbits. Science 1985; 228: 600-603.

21. Sladek JR, Gash DM (Eds), Neural Transplants: Development and Function. New York: Plenum, 1984; 454 pp.

22. Stelzner DL, Strauss JA. A quantitative analysis of frog optic nerve regeneration: Is retrograde ganglion cell death or collateral axonal loss related to selective re-innervation? J Comp Neurol 1986; 245: 83-106.

23. Turner JE, Blair JR. Newborn rat retinal cells transplanted into a retinal lesion site in adult host eyes. Dev Brain Res 1986; 26: 91-104.

24. Turner JE, Blair JR. Neonatal rat retinal grafts integrate into the lesion site of adult host retina. Invest Ophthalmol 1985; 26: 336.

25. Vidal-Sanz M, Bray GM, Villegas-Perez MP, Thanos $\mathrm{S}$, Aguayo AJ. Axonal regeneration and synapse formation in the superior colliculus by retinal ganglion cells in the adult rat. J Neurosci 1987; 7: 2894-2909.

26. Villegas-Perez MP, Vidal-Sanz M, Bray GM, Aguayo AJ. Influences of peripheral nerve grafts on the survival and regrowth of axotomized retinal ganglion cells in adult rats. J Neurosci 1988; 8: 265-280. 

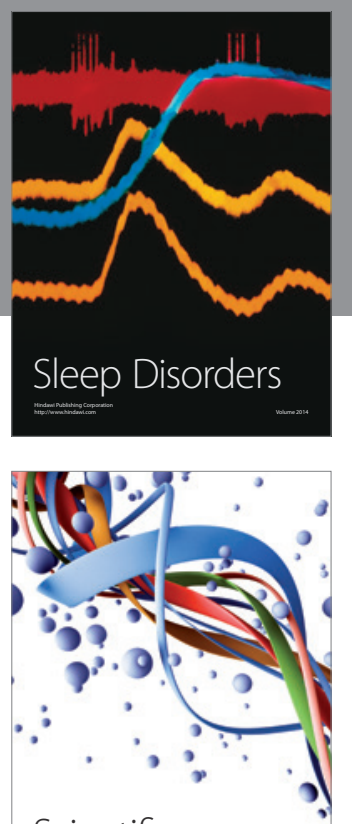

Scientifica
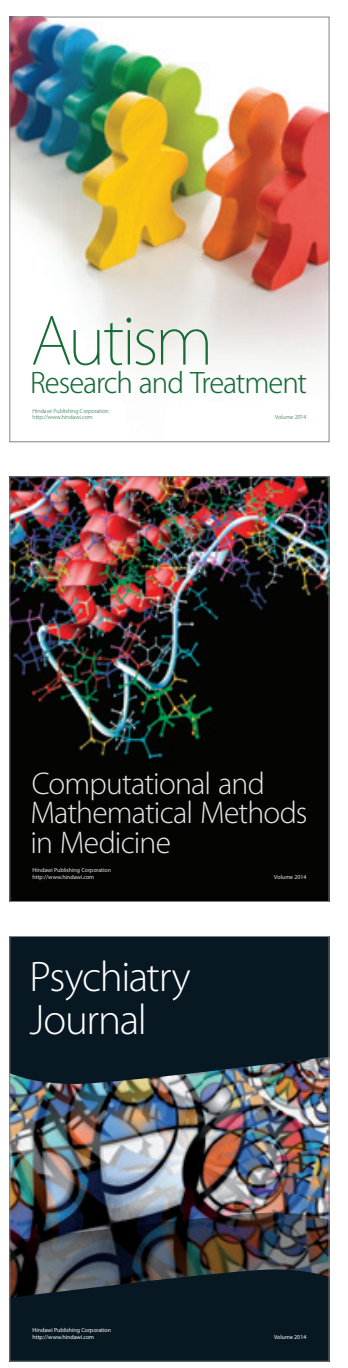
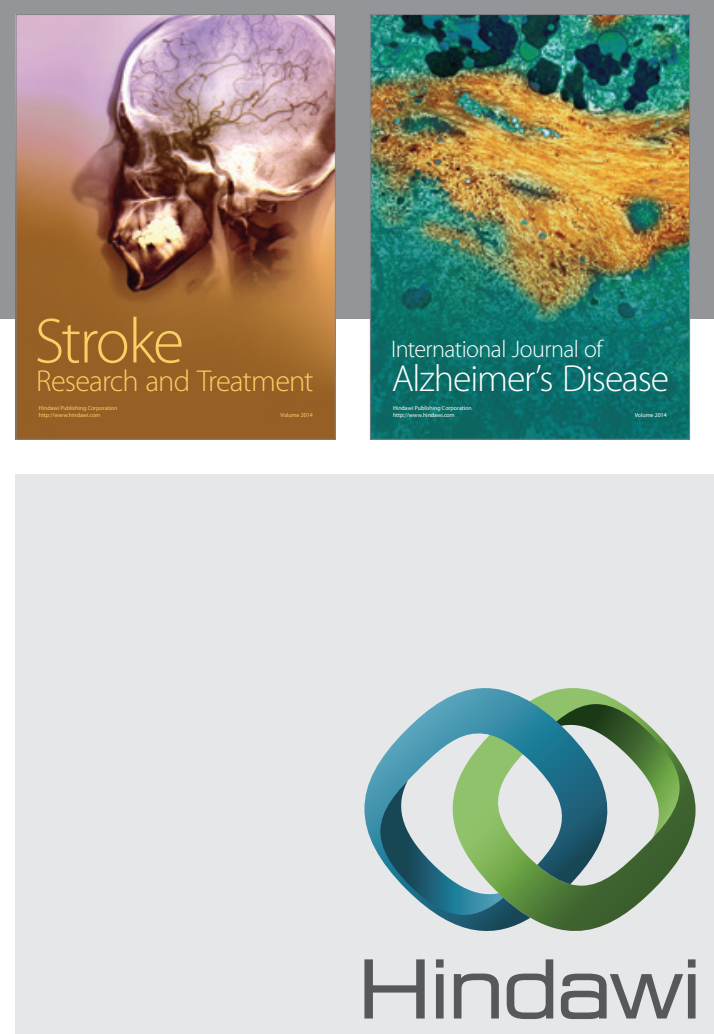

Submit your manuscripts at

http://www.hindawi.com
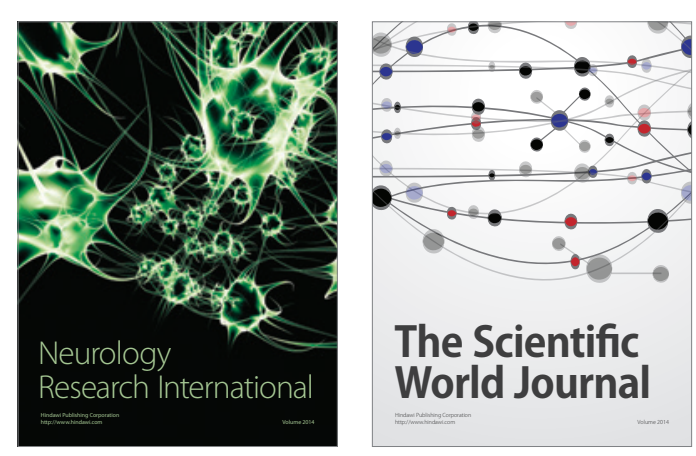

The Scientific World Journal

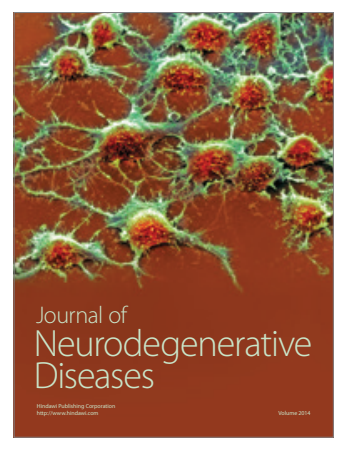

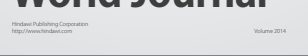

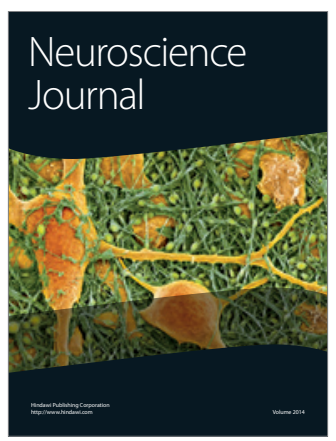

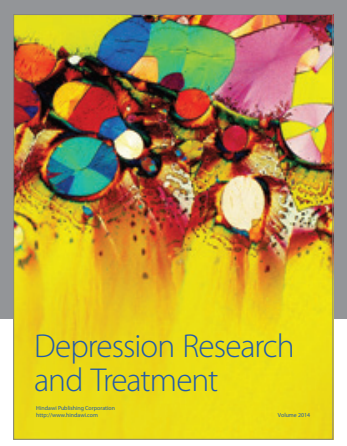
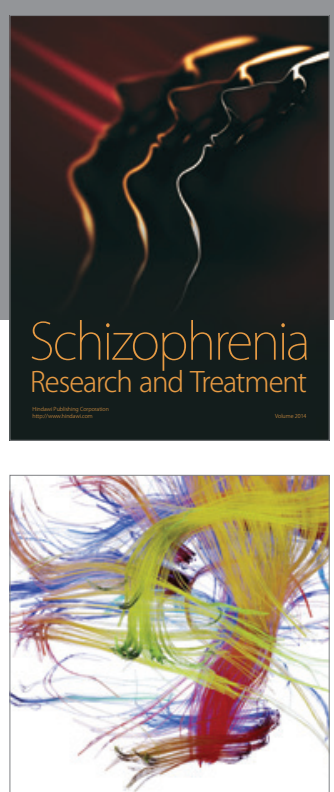

Brain Science

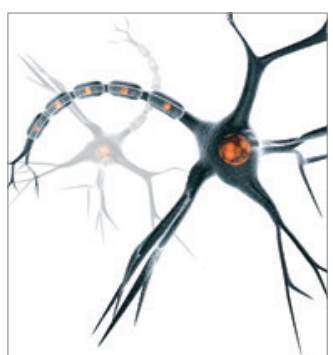

Neural Plasticity
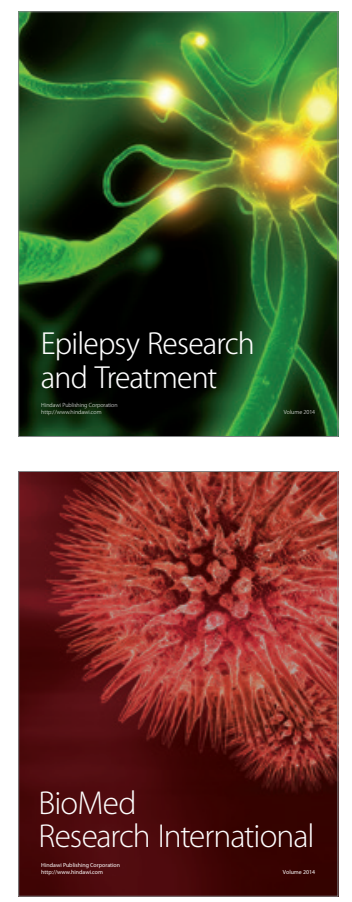

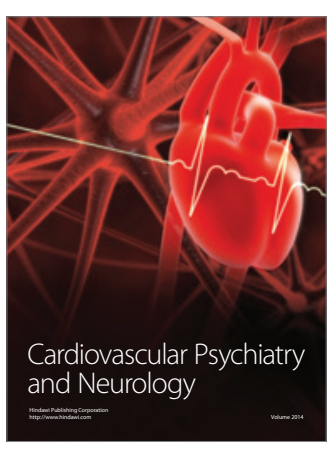

Parkinson's

Disease
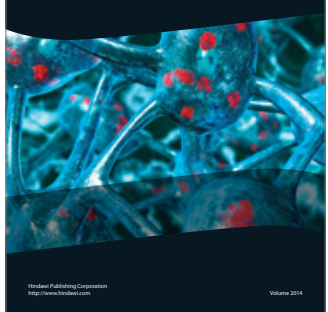